\title{
Musical Auditory Stimulation Influences Heart Rate Autonomic Responses to Endodontic Treatment
}

\author{
Milana Drumond Ramos Santana, ${ }^{1,2}$ Eli Carlos Martiniano, ${ }^{1,2}$ \\ Larissa Raylane Lucas Monteiro, ${ }^{1,2}$ Vitor E. Valenti, ${ }^{3}$ David M. Garner, ${ }^{1,4}$ \\ Isabel Cristina Esposito Sorpreso, ${ }^{1,5}$ and Luiz Carlos de Abreu ${ }^{1}$ \\ ${ }^{1}$ Laboratório de Delineamento de Estudos e Escrita Científica, Faculdade de Medicina do ABC, Santo André, SP, Brazil \\ ${ }^{2}$ Faculdade de Juazeiro do Norte, Juazeiro do Norte, CE, Brazil \\ ${ }^{3}$ Centro de Estudos do Sistema Nervoso Autônomo (CESNA), Departamento de Fonoaudiologia, \\ Faculdade de Filosofia e Ciências, UNESP, Marília, SP, Brazil \\ ${ }^{4}$ Cardiorespiratory Research Group, Department of Biological and Medical Sciences, \\ Faculty of Health and Life Sciences, Oxford Brookes University, Gipsy Lane, Oxford OX3 OBP, UK \\ ${ }^{5}$ Disciplina de Ginecologia do Departamento de Obstetrícia e Ginecologia, FMUSP, São Paulo, SP, Brazil
}

Correspondence should be addressed to Milana Drumond Ramos Santana; mildrumond@hotmail.com

Received 11 July 2016; Revised 20 October 2016; Accepted 17 November 2016; Published 15 January 2017

Academic Editor: Jeng-Ren Duann

Copyright (C) 2017 Milana Drumond Ramos Santana et al. This is an open access article distributed under the Creative Commons Attribution License, which permits unrestricted use, distribution, and reproduction in any medium, provided the original work is properly cited.

\begin{abstract}
We aimed to evaluate the acute effect of musical auditory stimulation on heart rate autonomic regulation during endodontic treatment. The study included 50 subjects from either gender between 18 and 40 years old, diagnosed with irreversible pulpitis or pulp necrosis of the upper front teeth and endodontic treatment indication. HRV was recorded 10 minutes before (T1), during (T2), and immediately (T3 and T4) after endodontic treatment. The volunteers were randomly divided into two equal groups: exposed to music (during T2, T3, and T4) or not. We found no difference regarding salivary cortisol and anxiety score. In the group with musical stimulation heart rate decreased in T3 compared to T1 and mean RR interval increased in T2 and T3 compared to T1. SDNN and TINN indices decreased in T3 compared to T4, the RMSSD and SD1 increased in T4 compared to T1, the SD2 increased compared to T3, and LF (low frequency band) increased in T4 compared to T1 and T3. In the control group, only RMSSD and SD1 increased in T3 compared to T1. Musical auditory stimulation enhanced heart rate autonomic modulation during endodontic treatment.
\end{abstract}

\section{Introduction}

Dental treatment has been reported by many patients as a situation generating stress and anxiety. One of the main elements that seem to interfere with the behavior of most individuals seeking dental care is the acceptance that they will be subjected to some kind of discomfort during the treatment [1-4].

In this context, the literature has investigated alternative therapies to improve stressful situations [5, 6]. Among alternative therapies, we may include the beneficial actions of musical therapy, which have been demonstrated as an alternative therapy for cardiovascular syndromes [7]. The use of relaxant music reduces the levels of heart rate, systolic blood pressure, and diastolic blood pressure [8].

Stress and anxiety are associated with the regulation of cardiac function by the autonomic nervous system (ANS), which allows us to evaluate the balance between the sympathetic and parasympathetic systems. In the heart, the ANS specifically influences heart rate and cardiac contractility. Furthermore, to increase or decrease of heart rate, ANS also regularly controls heart beats. The fluctuation of consecutive heart beats is analyzed through heart rate variability (HRV) [9-12].

Along these lines, the development of technical, scientific, and biological procedures in endodontic treatment achieves 
highly successful results. However, in spite of this, the treatment involves several stages ranging from anesthesia until obliteration of the root canal system. Also, the technique can be accomplished by basic technical operative steps which are prone to failure and various complications in its clinical progression [13].

Consequently, we believe that, during endodontic treatment, musical auditory stimulation may improve HRV. Knowledge of physiological responses involved in endodontic treatment is imperative because it provides a more appropriate treatment plan to the patient and provides greater safety clinically for the endodontist. Analysis of the ANS oscillations during endodontic procedure provides information, which can cause potential risks for cardiovascular system. Therefore, we investigated the acute effects of musical auditory stimulation on heart rate autonomic regulation during endodontic treatment.

\section{Method}

2.1. Population Study. Fifty subjects participated in this study with 13 females and 12 males in the music group and 12 females and 13 males in the control group. All participants aged between 18 and 40 years were examined. Everyone was diagnosed with irreversible pulpitis or pulp necrosis of the upper front teeth and endodontic treatment indication. The patients were divided into two equal groups: control group (patients who were submitted to standardized endodontic treatment) and music group (patients who were submitted to standardized endodontic treatment and were exposed to musical auditory stimulation). The sample was selected from endodontic treated patients undergoing the root canal procedure. All volunteers were informed about the procedures and objectives of the study and, after agreeing, they signed an informed consent form which was confidential. The study was approved by the Ethics Committee in Research of our University (Protocol number 993.350).

2.2. Exclusion Criteria. We considered the following as exclusion criteria: smokers; individuals with cardiorespiratory, neurological disorders and other reported impairments that prevented the subject from performing the procedures and treatment with drugs that influence cardiac autonomic regulation; patients who have undergone previous root canal treatment; women between 10-15 and 20-25 days after the first day of the menstrual cycle in order to avoid influence of luteal and follicular phase; pregnant women; and subjects who reported hearing disorders.

2.3. Initial Evaluation. Prior to the endodontic treatment the following information was collected: age, gender, mass, height, and body mass index (BMI). Anthropometric measurements were obtained according to the recommendations described by Lohman [14]. Mass was measured on a digital scale (W 200/5, Welmy, Brazil) with a precision of $0.1 \mathrm{~kg}$ and height using a stadiometer (ES 2020 Sanny, Brazil). BMI was calculated using the following formula: mass (kg)/height $(\mathrm{m})^{2}$.
2.4. Experimental Protocols. Data collection was undertaken in a room with temperature between $21^{\circ} \mathrm{C}$ and $25^{\circ} \mathrm{C}$ and humidity between $50 \%$ and $60 \%$. The volunteers were instructed to not ingest alcohol, caffeine, or other ANS stimulants for 24 hours before the evaluation. The volunteers were instructed to remain at rest and avoid talking during the collection.

Primarily, the blood pressure (BP) measurement was implemented. Subsequently, the saliva collection for salivary cortisol measurement was undertaken. Following this the analysis of the protocol of cardiac modulation during endodontic treatment was assumed.

2.5. Blood Pressure Measurement. Systolic (SBP) and Diastolic Blood Pressure (DBP) measurements were performed using an aneroid sphygmomanometer $\left(\operatorname{Tycos}^{\circledR}\right)$ and stethoscope $\left(\right.$ Sprague $\left.{ }^{\circledR}\right)$. BP was measured before and after the endodontic treatment.

2.6. Measurement of Salivary Cortisol. We did not induce salivation to collect saliva samples; we collected salivary cortisol samples before and after endodontic treatment between 14:00 and 17:00 by the same researcher. The volunteers were told to not eat or drink one hour before collecting saliva and wash their mouths with water. The Salivette ${ }^{\circledR}$ tubes were refrigerated until analysis of the material.

Data assembly was performed using a Salivette tube consisting of a plastic tube that contains a highly absorbent cotton roll, maintained in the mouth for about three minutes. Then, it was placed inside the collector plastic tube. After collecting all the Salivette tubes, samples were centrifuged for 20 minutes at $2800 \mathrm{rpm}$ at a temperature of $4^{\circ} \mathrm{C}$. During centrifugation, saliva passed the cylindrical swab medium through the cavity in the bottom of the drop tube to the clean centrifuge tube. Mucus and airborne particles were captured in the conical tip of the tube, allowing for easy decanting of clarified saliva.

Following centrifugation, the saliva was transferred to an Eppendorf type tube with lid and stored in a freezer at $-20^{\circ} \mathrm{C}$ until the time when it was inspected. The material was cooled, ensured by the use of dry ice, Styrofoam, and a thermal bag. The samples were processed using the electrochemiluminescence method.

2.7. HRV Analysis during Endodontic Treatment. HRV was recorded in the first session of endodontic treatment at four intervals:

(i) 10 minutes before the beginning of root canal therapy session (T1)

(ii) The initial 10 minutes after the application of local anesthetic (T2)

(iii) The total duration of endodontic treatment (T3)

(iv) 30 minutes after the endodontic treatment termination (T4)

After the initial assessment, we placed the heart rate receiver Polar RS800CX (Polar Electro, Finland) on the chest of the volunteers at the distal third of the sternum region and 
strapped at the wrist. After placing the strap and the monitor, the protocol was initiated.

2.8. Protocol of Endodontic Treatment. The endodontic treatment was performed by the same dentist. We describe below all the steps of endodontic treatment:

(i) Inferior alveolar nerve anesthesia (2\% DFL ${ }^{\circledR}$ ALPHACAINE)

(ii) Decayed tissue removal and access to the pulp chamber (spherical diamond burs numbers 1012, 1014, and 1015, KG Sorensen ${ }^{\circledR}$ ) compatible with the size of the pulp chamber, mounted on high speed turbine, aircooled water, and Endo Z bur (Les Fils d' August Mailefer SA)

(iii) The operating field isolation with rubber dam

(iv) Chemical-mechanical preparation of root canals (Hand files Flexofile Dentsply ${ }^{\circledR}$ and sodium hypochlorite $2.5 \%$ )

(v) Channel drying (Dentsply paper cones)

(vi) Intracanal medication (SS White ${ }^{\circledR}$ )

(vii) Cotton ball laying on channel input

(viii) Interim sealing $\left(\right.$ IRM $\left.^{\circledR}\right)$

2.9. The Effect of Music on HRV Analysis. The volunteers were randomly divided into two equal groups $(n=25)$, in which a group performed endodontic treatment while listened to music during T2, T3, and T4 periods and the other group was not exposed to musical auditory stimulation. The music played was "Träumerei" of Kinderszenen Op. 15-7 and was conveyed through a mp4 player (Sony ${ }^{\circledR}$ NWZ-E473) to an earphone. The control group remained with the earphone turned-off.

2.10. HRV Analysis. HRV analysis followed guidelines from the aforementioned Task Force [15]. The RR intervals were recorded by the portable RS800CX heart rate (HR) monitor with a sampling rate of $1000 \mathrm{~Hz}$. They were then downloaded to the Polar Precision Performance program (v. 3.0, Polar Electro, Finland). The software enabled the visualization of HR and the extraction of a cardiac period (RR interval) file in "txt" format. Subsequently, digital filtering was complemented with manual filtering for the elimination of premature ectopic beats and artefacts; the final $256 \mathrm{RR}$ intervals of each period were applied for the data analysis, because this was the period in which the subjects were stable.

Digital filtering was based on an algorithm in the Polar Protrainer software (Polar Electro, Finland). The algorithm intends to detect all measurement errors through analyzing median and moving average based filtering methods. The criteria adapt to the RR interval to be verified; if RR interval is very stable, relatively small peaks will be interpreted as errors. However, if RR interval includes very large variability, the criteria will be looser. Very small errors are not corrected. In the correction phase, the algorithm computes several more matching "candidates" to substitute the detected errors. The algorithm identifies the difference between previous and the next accepted RR interval and then makes the series of corrected values to follow the same differential coefficient. The special property of the error correction algorithm maintains the total time of the recording: that is, the sum of RR intervals is exactly the same as the elapsed time (http://support.polar.com/en/support/tips/How_R-R_Data is_Filtered).

Manual filtering was performed by using Microsoft $^{\circledR}$ Excel ${ }^{\circledR}$ 2013, it was based on visual inspection that detect artefacts and ectopic beats.

Only series with more than $95 \%$ sinus rhythm were included in the study; we excluded records with a percentage of artefacts higher than 5\% [11, 16]. For calculation of the linear indices we applied the HRV analysis software (Kubios HRV ${ }^{\circledR}$ v.1.1 for Windows, Biomedical Signal Analysis Group, Department of Applied Physics, University of Kuopio, Finland) [17] (see Supplement files).

2.11. Geometric Indices of HRV. The geometric indices of HRV included quantitative analysis of the Poincare plot (SD1: standard deviation of the instantaneous variability of the beat-to-beat heart rate; SD2: standard deviation of long-term continuous RR interval variability and SD1/SD2 ratio) and time domain RRtri (triangular index) and TINN (triangular interpolation of RR intervals) indices. Significance of the abovementioned indices was previously described $[15,18]$.

\section{Statistical Analysis}

To determine the sample size a priori knowledge was needed, based on Moreno et al. [19] findings for SDNN (standard deviation of all normal RR intervals), which represents the activity of both ANS branches, indicating the overall behavior of HRV [11]. A sample size of 18 participants per group was stipulated by a test of hypothesis (two-tailed), with $5 \%$ level of significance and $80 \%$ power.

For comparison between individuals of the same group, it was necessary to assess data normality by the Shapiro-Wilk test. When normal distribution was achieved, we applied the unpaired Student's $t$-test and ANOVA for repeated measures followed by Bonferroni posttest. In circumstances where the normal distribution was unaccepted, the Mann-Whitney test and the Friedman test followed by Dunn's posttest was applied. Dissimilarities in these tests were considered statistically significant when the value of " $p$ " is less than 0.05 . The statistical program applied was GraphPad Software StatMate 2:00 version for Windows, GraphPad Software, San Diego, California, USA.

\section{Results}

After applying the normality test we checked that all distributions evaluated were parametric. In this sense, data were presented as mean and standard deviation.

Table 1 displays anthropometric data including age, height, mass, and BMI of volunteers from both groups.

In the control group, we noted that RR interval was increased during endodontic treatment (T3) compared to before endodontic procedures (T1). RMSSD (root-mean square of 
TABLE 1: Age, height, mass, and BMI of volunteers.

\begin{tabular}{|c|c|c|c|}
\hline \multirow{2}{*}{ Variable } & \multicolumn{2}{|c|}{ Mean \pm standard-deviation } & \multirow{2}{*}{$p$} \\
\hline & Control & Music & \\
\hline $\begin{array}{l}\text { Age (years) } \\
\text { (min-max) }\end{array}$ & $\begin{array}{c}29.16 \pm 6.99 \\
(18-40)\end{array}$ & $\begin{array}{c}28.61 \pm 6.88 \\
\quad(19-40)\end{array}$ & 0.7628 \\
\hline $\begin{array}{l}\text { Height }(\mathrm{m}) \\
(\min -\max )\end{array}$ & $\begin{array}{c}1.61 \pm 0.08 \\
(1.36-1.8)\end{array}$ & $\begin{array}{c}1.6 \pm 0.08 \\
(1.47-1.72)\end{array}$ & 0.6512 \\
\hline $\begin{array}{l}\text { Mass (kg) } \\
(\min -\max )\end{array}$ & $\begin{array}{c}65.72 \pm 12.03 \\
(43.3-91.3)\end{array}$ & $\begin{array}{c}63.53 \pm 10.1 \\
\quad(56-91)\end{array}$ & 0.4704 \\
\hline $\begin{array}{l}\operatorname{BMI}\left(\mathrm{kg} / \mathrm{m}^{2}\right) \\
(\min -\max )\end{array}$ & $\begin{array}{c}26.14 \pm 6.44 \\
(18.59-47.43)\end{array}$ & $\begin{array}{c}24.82 \pm 3.85 \\
(18.72-33.89)\end{array}$ & 0.6176 \\
\hline
\end{tabular}

$\mathrm{m}$ : meters; kg: kilograms; BMI: height and body mass index; min-max: minimum-maximum.

differences between adjacent normal RR intervals) and SD1 indices were also increased during endodontic treatment (T3) compared to before endodontic procedures ( $\mathrm{Tl}$ ) (Table 2).

Regarding the groups subjected to musical auditory stimulation during the endodontic treatment, we observed that DAP increased after the procedures (T4). HR decreased while RR interval decreased during endodontic treatment (T3) compared to before treatment (T1) (Table 3).

Regarding HRV analysis, SDNN, TINN, and SD2 indices were weakened during endodontic treatment (T3) compared to after treatment (T4). Moreover, RMSSD and SD1 were increased after treatment (T4) compared to before treatment (T1) (Table 3).

Regarding spectral analysis, LF in absolute units was increased after treatment (T4) compared to before (T1) and during treatment (T3) (Table 3).

In our study, we found a decrease in the SDNN, SD2, and LF indices during endodontic treatment with musical stimulation compared to after treatment. Those indices correspond to overall HR modulation [15].

We also compared HRV indices between both groups at all instants. SDNN $(p=0.01)$, TINN $(p=0.01)$, and SD2 $(p=0.002)$ were increased in the control group during T3 with no changes in T1, T2, and T4. TINN was higher in T1 $(p=0.04)$ and T3 $(p=0.001)$ in the control group with no changes in T2 and T4. The LF/HF ratio was increased in T1 $(p=0.04)$ and $\mathrm{T} 2(p=0.02)$ in the control group with no changes in T3 and T4.

There was no significant difference between groups for mean RR interval, RMSSD, pNN40, SD1, LF $\left(\mathrm{ms}^{2}\right)$, LF (nu), HF $\left(\mathrm{ms}^{2}\right)$, and HF (nu) LF/HF ratio during T1, T2, T3, and T4.

\section{Discussion}

This study assessed the acute effect of musical auditory stimulation on HRV and salivary cortisol during endodontic treatment. Accordingly, it was found that the musical auditory stimulus improved heart rate autonomic responses induced by endodontic treatment. There were no significant changes in cortisol levels, which indicates the involvement of ANS on this mechanism.
During endodontic treatment, the patient is commonly presented in a stressful condition [1]. The physical or psychological stress stimulates the hypothalamus to synthesize corticotrophin releasing hormone, which then stimulates the pituitary gland into synthesizing the adrenocorticotropic hormone, which flows via the bloodstream to the adrenal glands and stimulate the synthesis of cortisol, the "stress hormone" [4]. Simultaneously there is activation of the sympathetic nervous system and this releases catecholamines (norepinephrine and epinephrine), which then trigger increased $\mathrm{HR}$, myocardial contraction, cardiac output, and arterial pressure [20].

Equally, we failed to find significant changes in cortisol levels after endodontic treatment in control and music group. Nevertheless, there is a trend of declination after treatment in the group exposed to musical auditory stimulation.

Regarding the effects of stress, anxiety, and depression on modulation of the ANS, Langewitz et al. [21] evaluated the effect of mental stress on HRV and they observed a decrease of HRV. According to Morino et al. [22], the HRV spectral analysis is advantageous in the evaluation of tension and anxiety.

The study of Matsumura et al. [23] assessed changes in blood pressure and HRV during dental surgery. It was verified that elderly patients had a further severe increase in blood pressure during dental surgeries compared to younger patients and that HRV during dental surgery differs between younger and elderly patients. Miura et al. [12] evaluated the changes in blood pressure, pulse rate, and HRV during dental surgery in hypertensive patients. The authors concluded that suppression of cardiac sympathetic nervous system during dental surgery may weaken responses in patients with hypertension.

Moreover, Santana et al. [24] observed that during endodontic treatment HRV is diminished. Furthermore, after application of the local anesthetic the HRV increases due to cardiac parasympathetic predominance. Like this, we aimed to verify the effects of an alternative therapy to improve HRV responses to endodontic treatment.

In our study, we found a decrease in the SDNN, SD2, and LF indices during endodontic treatment with musical stimulation compared to after treatment. Those indices correspond to overall HR modulation [15].

The auditory stimulation with music influences the cardiovascular system, since there is a correlation between the noise intensity and sympathovagal balance. It is hypothesized that dopamine released in the striatal system induced by melodic and cheerful songs is involved in autonomic regulation $[7,25]$. In this way, music is capable of improving autonomic regulation of heart rate.

Conferring to da Silva et al. [26] classical music increases the parasympathetic modulation. Riganello et al. [27] evaluated the HRV and its correlation with musical stimulation of different complexities in healthy subjects and with disorders of perception. The results illustrated that the musical stimulus improved the autonomic response of patients with perception disorders.

Some studies illustrated a correlation between parasympathetic heart rate regulation (HF: high frequency band) and 
TABLE 2: Mean and standard deviation of SBP, DBP, and cortisol in T1 and T4, heart rate (HR), RR interval, and linear indices in T1, T2, T3, and T4 in the volunteers without auditory stimulus.

\begin{tabular}{|c|c|c|c|c|c|}
\hline Variable & $\mathrm{T} 1$ & $\mathrm{~T} 2$ & T3 & $\mathrm{T} 4$ & Value $p$ \\
\hline SAP (mmHg) & $117.2 \pm 17.68$ & - & - & $118.4 \pm 17$ & 0.7033 \\
\hline DAP (mmHg) & $77.6 \pm 12$ & - & - & $79.2 \pm 9.96$ & 0.3075 \\
\hline Cortisol (ug/dl) & $0.23 \pm 0.13$ & - & - & $0.4 \pm 0.77$ & 0.1334 \\
\hline HR (bpm) & $90.5 \pm 20.96$ & $89.42 \pm 29.93$ & $78.81 \pm 14.1$ & $86.69 \pm 30.35$ & 0.1851 \\
\hline $\mathrm{RR}$ (ms) & $695.98 \pm 136.66^{*}$ & $731.06 \pm 184.17$ & $786.92 \pm 126.75$ & $753.5 \pm 183.29$ & $p<0.0001$ \\
\hline $\operatorname{SDNN}(\mathrm{ms})$ & $45.11 \pm 17.44$ & $45.78 \pm 30.28$ & $54.08 \pm 25.87$ & $44.86 \pm 16.98$ & 0.4717 \\
\hline RMSSD (ms) & $27.68 \pm 17^{*}$ & $33.1 \pm 24.54$ & $44.76 \pm 25.49$ & $34.94 \pm 22.62$ & 0.0251 \\
\hline pNN50 (ms) & $10.69 \pm 13.3$ & $15.86 \pm 18.49$ & $22.76 \pm 17.95$ & $17.4 \pm 17.67$ & 0.0620 \\
\hline RRtri (ms) & $9.86 \pm 4.83$ & $9.41 \pm 5.58$ & $11.66 \pm 6.39$ & $9.76 \pm 5.2$ & 0.1472 \\
\hline TINN (ms) & $183.8 \pm 74.76$ & $163.8 \pm 101.79$ & $212.2 \pm 101.43$ & $166 \pm 73.47$ & 0.0626 \\
\hline $\mathrm{SD} 1$ (ms) & $19.66 \pm 12.05^{*}$ & $23.5 \pm 17.39$ & $31.8 \pm 18.15$ & $24.83 \pm 16.06$ & 0.0240 \\
\hline $\mathrm{SD} 2(\mathrm{~ms})$ & $59.78 \pm 23.89$ & $59.33 \pm 40.21$ & $68.92 \pm 33.47$ & $57.26 \pm 21.31$ & 0.6685 \\
\hline $\mathrm{LF}\left(\mathrm{ms}^{2}\right)$ & $598.96 \pm 499.28$ & $608.12 \pm 739.56$ & $870.43 \pm 915.79$ & $872.06 \pm 910.3$ & 0.3427 \\
\hline $\mathrm{LF}(\mathrm{nu})$ & $64.06 \pm 20.13$ & $62.27 \pm 22.72$ & $57.24 \pm 16.34$ & $62.69 \pm 20.39$ & 0.8741 \\
\hline $\mathrm{HF}\left(\mathrm{ms}^{2}\right)$ & $459.37 \pm 669.23$ & $491.84 \pm 706.79$ & $784.87 \pm 960.68$ & $529.4 \pm 631.87$ & 0.1104 \\
\hline $\mathrm{HF}(\mathrm{nu})$ & $35.78 \pm 20.06$ & $37.52 \pm 22.64$ & $42.56 \pm 16.33$ & $37.18 \pm 20.34$ & 0.4329 \\
\hline $\mathrm{LF} / \mathrm{HF}$ & $3.42 \pm 4.02$ & $3.66 \pm 4.47$ & $2.23 \pm 3.26$ & $3.08 \pm 3.07$ & 0.8741 \\
\hline
\end{tabular}

${ }^{*} p<0,05$ : versus T3. ms: milliseconds; bpm: beat per minute; $\mathrm{mmHg}$ : millimeters of mercury; ug/dl: micrograms per deciliter; nu: standard unit; $\mathrm{ms}{ }^{2}$ : absolute unity; SDNN: standard deviation of all normal RR intervals; pNN50: percentage of adjacent RR intervals lasting difference greater than 50 milliseconds; RMSSD: square root of the mean square of differences between adjacent normal RR intervals; LF: low frequency; HF: high frequency; LF/HF: ratio low frequency/high frequency; RRtri, triangular index; TINN, triangular interpolation of RR intervals; SDI: standard deviation of the instantaneous variability of the beat-to-beat heart rate; SD2: standard deviation of long-term continuous RR interval variability; SD1/SD2 ratio: ratio between the short- and long-term variation of the RR intervals.

TABLE 3: Mean and standard deviation of SBP, DBP, and cortisol in T1 and T4, heart rate, RR interval, and linear indices in T1, T2, T3, and T4 in subjects with auditory stimulus.

\begin{tabular}{|c|c|c|c|c|c|}
\hline Variable & $\mathrm{T} 1$ & $\mathrm{~T} 2$ & T3 & $\mathrm{T} 4$ & Value $p$ \\
\hline SAP (mmHg) & $116.15 \pm 14.71$ & - & - & $117.3 \pm 11.85$ & 0.6085 \\
\hline DAP (mmHg) & $78.46 \pm 10.07$ & - & - & $82.3 \pm 7.64$ & 0.0288 \\
\hline Cortisol (ug/dl) & $0.27 \pm 0.17$ & - & - & $0.24 \pm 0.15$ & 0.0577 \\
\hline HR (bpm) & $82.25 \pm 13.08$ & $79.3 \pm 15.02$ & $76.73 \pm 12.09^{*}$ & $114.55 \pm 177.84$ & 0.0066 \\
\hline $\mathrm{RR}(\mathrm{ms})$ & $747.93 \pm 125.7$ & $787.08 \pm 151.53^{*}$ & $804.03 \pm 135.94^{*}$ & $779.63 \pm 122.1$ & 0.0014 \\
\hline SDNN (ms) & $40.34 \pm 14.47$ & $45.2 \pm 16.35$ & $38.51 \pm 16.23^{* *}$ & $46.9 \pm 15.09$ & 0.0237 \\
\hline RMSSD (ms) & $34.66 \pm 18.01^{* *}$ & $41.92 \pm 22.53$ & $37.13 \pm 21.71$ & $39.65 \pm 19.15$ & 0.0240 \\
\hline pNN50 (ms) & $15.39 \pm 16.05$ & $21.69 \pm 19.42$ & $16.84 \pm 18.3$ & $18.09 \pm 15.11$ & 0.1033 \\
\hline RRtri (ms) & $8.31 \pm 2.48$ & $8.53 \pm 2.83$ & $8.36 \pm 3.09$ & $8.7 \pm 1.84$ & 0.6246 \\
\hline TINN (ms) & $152.3 \pm 56.46$ & $156.73 \pm 66.75$ & $138.46 \pm 67.03^{* *}$ & $171.53 \pm 53.41$ & 0.0320 \\
\hline $\mathrm{SD} 1$ (ms) & $24.67 \pm 12.8^{* *}$ & $29.89 \pm 16.08$ & $26.45 \pm 15.42$ & $28.23 \pm 13.61$ & 0.0310 \\
\hline $\mathrm{SD} 2$ (ms) & $51 \pm 17.61$ & $55.34 \pm 19.97$ & $46.95 \pm 18.64^{* *}$ & $59.74 \pm 17.94$ & 0.0336 \\
\hline $\operatorname{LF}\left(\mathrm{ms}^{2}\right)$ & $286 \pm 61.04^{* *}$ & $576.88 \pm 423.06$ & $529 \pm 592.94^{* *}$ & $850.92 \pm 615.42$ & 0.0004 \\
\hline $\mathrm{LF}(\mathrm{nu})$ & $57.77 \pm 16.33$ & $54.25 \pm 18.99$ & $49.14 \pm 23.26$ & $60.19 \pm 15.09$ & 0.0982 \\
\hline $\mathrm{HF}\left(\mathrm{ms}^{2}\right)$ & $470.57 \pm 466.43$ & $539.38 \pm 431.14$ & $471.26 \pm 466.46$ & $654.69 \pm 647.14$ & 0.3741 \\
\hline HF (nu) & $41.94 \pm 16.26$ & $45.5 \pm 18.95$ & $50.56 \pm 23.15$ & $39.64 \pm 15.07$ & 0.1007 \\
\hline $\mathrm{LF} / \mathrm{HF}$ & $1.89 \pm 1.66$ & $1.73 \pm 1.46$ & $1.89 \pm 2.45$ & $2.1 \pm 1.97$ & 0.1391 \\
\hline
\end{tabular}

${ }^{*} p<0,05$ : versus T1; ${ }^{* *} p<0,05$ : versus T4. ms: milliseconds; bpm: beat per minute; mmHg: millimeters of mercury; ug/dl: micrograms per deciliter; $\mathrm{nu}$ : standard unit; $\mathrm{ms}^{2}$ : absolute unity; SDNN: standard deviation of all normal RR intervals; pNN50: percentage of adjacent RR intervals lasting difference greater than 50 milliseconds; RMSSD: square root of the mean square of differences between adjacent normal RR intervals; LF: low frequency; HF: high frequency; LF/HF: ratio low frequency/high frequency; RRtri, triangular index; TINN, triangular interpolation of RR intervals; SDI: standard deviation of the instantaneous variability of the beat-to-beat heart rate; SD2: standard deviation of long-term continuous RR interval variability; SD1/SD2 ratio: ratio between the short- and long-term variation of the RR intervals. 
music [28, 29]. Supplementary studies suggested that the music decreases excitatory activation of the parasympathetic nervous system in healthy subjects [28] and heavy metal sharply decreases HRV [23]. do Amaral et al. [25] described that auditory stimulation with baroque music did not affect heart rate control. Yet, higher sound intensity of heavy metal reduced the global component of HRV.

Also, reduction of the LF index was observed in the study of da Silva et al. [26], which evaluated the acute effects of heavy metal and baroque musical auditory stimulation on HRV in healthy males. The authors noted that the LF band, in both absolute and normalized standard units, was lessened during exposure to both styles of music compared to the control groups. In the same study, there was a reduction in SDNN index during heavy metal music.

We reported that the TINN index was also decreased during endodontic treatment in the music group. Previously a study [25] illustrated that auditory stimulation with heavy metal music caused a reduction of TINN index in females. Nevertheless, in another study [30] no change was observed in relation to TINN during the musical stimulation with heavy metal in males.

Founded on our data, RMSSD and SD1 indices increased after endodontic treatment compared to before treatment. Our data points to a beneficial elevation in parasympathetic heart rate regulation induced by music during endodontic treatment. Ferreira et al. [31] testified increases in SDNN, RMSSD, pNN50 (percentage of adjacent RR intervals with a difference of duration greater than $50 \mathrm{~ms}$ ), and LF $\left(\mathrm{ms}^{2}\right)$ after stimulation with heavy metal music in healthy females. The authors also found that SDNN, LF ( $\mathrm{ms}^{2}$ and $\mathrm{nu}$ ), and the LF/HF ratio increased while the HF index (nu) decreased after exposure to classical baroque music. The abovementioned study supports our results.

In this study, there was deficiency of significance of salivary cortisol before and after endodontic treatment. In this context, we indicate that music operated through ANS and not via hypothalamic-pituitary axis due to the short period of endodontic treatment. Nonetheless, there was a trend $(p=0.0577)$ to reduce cortisol levels in the music group. Hence, we suggest that a more prolonged time of exposure to music may influence cortisol levels.

Previously, a study stated that music has a beneficial effect in controlling anxiety to dental treatment. The authors found significant differences in salivary cortisol levels, systolic and diastolic pressure, HR, body temperature, and stimulated salivary flow for the group treated with musical treatment [32]. Likewise, our findings reinforce the beneficial use of music during endodontic treatment.

Mehr et al. [33] assessed techniques of music therapy in different dental specialties, wherein 81 patients reported the dental treatment as unpleasant and then were driven to a discrete music therapy. After each dental treatment session, both patients and the dentist responded to a questionnaire. In conclusion, the authors encouraged music therapy in dental treatment, which supports our findings here.

According to our results, we reported a significant statistical increase of LF and a simultaneous decrease of SDNN between T1 and T3 during auditory stimulus; it indicated that the music induced excitatory effects on HRV. A previous study assisted us to explain this response. Roque et al. [34] observed that relaxant baroque music slightly decreased global HRV. Taken together, we believe that the musical auditory stimulation used in our study may have caused a similar reaction on HRV.

Some facts are worth considering. The age range was wide (18 to 40 years). Conversely, exclusion criteria helped to ensure regulatory standards regarding the sample selection. Factors such as age, gender, anthropometric characteristics, and health conditions have been shown to influence physiological reactions [35]. We investigated the patients in the first session of endodontic treatment in order to avoid prior familiarity with the endodontic treatment.

\section{Conclusions}

Musical auditory stimulation acutely improved heart rate autonomic regulation during endodontic treatment. Our results encourage the use of relaxant music during endodontic treatment in order to lessen cardiovascular responses.

\section{Competing Interests}

The authors declare that there is no conflict of interests regarding the publication of this paper.

\section{Acknowledgments}

This study received financial support from FAPESP (Process 2012/01366-6).

\section{References}

[1] M. D. R. Santana, A. C. A. De Souza, L. C. De Abreu, and V. E. Valenti, "Association between oral variables and heart rate variability," International Archives of Medicine, vol. 6, article 49, 2013.

[2] R. F. Possobon, A. B. A. Moraes, Á. L. Costa Junior, and G. M. B. Ambrosano, "O comportamento de crianças durante atendimento odontológico," Psicologia: Teoria e Pesquisa, vol. 19, no. 1, pp. 59-64, 2003.

[3] R. Poulton, W. M. Thomson, R. H. Brown, and P. A. Silva, "Dental fear with and without blood-injection fear: implications for dental health and clinical practice," Behaviour Research and Therapy, vol. 36, no. 6, pp. 591-597, 1998.

[4] S. Akyuz, S. Pince, and N. Hekim, "Children's stress during a restorative dental treatment: assessment using salivary cortisol measurements," Journal of Clinical Pediatric Dentistry, vol. 20, no. 3, pp. 219-223, 1996.

[5] L.-L. Wu, Y. Liu, C. Yan, Y. Pan, J.-F. Su, and W.-K. Wu, "Antidepressant-like effects of fractions prepared from DanzhiXiaoyao-San decoction in rats with chronic unpredictable mild stress: effects on hypothalamic-pituitary-adrenal axis, arginine vasopressin, and neurotransmitters," Evidence-Based Complementary and Alternative Medicine, vol. 2016, Article ID 6784689, 11 pages, 2016. 
[6] L. Rapolienė, A. Razbadauskas, J. Sąlyga, and A. Martinkenas, "Stress and fatigue management using balneotherapy in a shorttime randomized controlled trial," Evidence-Based Complementary and Alternative Medicine, vol. 2016, Article ID 9631684, 10 pages, 2016.

[7] V. E. Valenti, H. L. Guida, C. B. D. M. Monteiro et al., "Relationship between cardiac autonomic regulation and auditory mechanisms: importance for growth and development," Journal of Human Growth and Development, vol. 23, no. 1, pp. 94-98, 2013.

[8] Y.-L. Ko and P.-C. Lin, "The effect of using a relaxation tape on pulse, respiration, blood pressure and anxiety levels of surgical patients," Journal of Clinical Nursing, vol. 21, no. 5-6, pp. 689697, 2012.

[9] M. Malik and A. J. Camm, "Components of heart rate variability-what they really mean and what we really measure," The American Journal of Cardiology, vol. 72, no. 11, pp. 821-822, 1993.

[10] V. E. Valenti, “The recent use of heart rate variability for research," Journal of Human Growth and Development, vol. 25, no. 2, pp. 137-140, 2015.

[11] L. C. M. Vanderlei, C. M. Pastre, R. A. Hoshi, T. D. de Carvalho, and M. F. de Godoy, "Basic notions of heart rate variability and its clinical applicability," Brazilian Journal of Cardiovascular Surgery, vol. 24, no. 2, pp. 205-217, 2009.

[12] K. Miura, K. Matsumura, Y. Nakamura, H. Kurokawa, M. Kajiyama, and Y. Takata, "Suppression of cardiac sympathetic nervous system during dental surgery in hypertensive patients," Hypertension Research, vol. 23, no. 3, pp. 207-212, 2000.

[13] C. Estrela, Endodontic Science, Artes Médicas, São Paulo, Brazil, 2004.

[14] T. G. Lohman, Anthropometric Standardization Reference Manual, Human Kinetics Books, Champaign, Ill, USA, 1988.

[15] Task Force of the European Society of Cardiology and the North American Society of Pacing and Electrophysiology, "Heart rate variability: standards of measurement, physiological interpretation and clinical use," Circulation, vol. 93, no. 5, pp. 1043-1065, 1996.

[16] B. S. Tavares, G. de Paula Vidigal, D. M. Garner, R. D. Raimundo, L. C. de Abreu, and V. E. Valenti, "Effects of guided breath exercise on complex behaviour of heart rate dynamics," Clinical Physiology and Functional Imaging, 2016.

[17] J.-P. Niskanen, M. P. Tarvainen, P. O. Ranta-Aho, and P. A. Karjalainen, "Software for advanced HRV analysis," Computer Methods and Programs in Biomedicine, vol. 76, no. 1, pp. 73-81, 2004.

[18] M. P. Tulppo, T. H. Makikallio, T. Seppanen, R. T. Laukkanen, and H. V. Huikuri, "Vagal modulation of heart rate during exercise: effects of age and physical fitness," American Journal of Physiology-Heart and Circulatory Physiology, vol. 274, no. 2, pp. H424-H429, 1998.

[19] I. L. Moreno, C. M. Pastre, C. Ferreira, L. C. de Abreu, V. E. Valenti, and L. C. M. Vanderlei, "Effects of an isotonic beverage on autonomic regulation during and after exercise," Journal of the International Society of Sports Nutrition, vol. 10, no. 1, article no. 2, 2013.

[20] A. E. Aubert, B. Seps, and F. Beckers, "Heart rate variability in athletes," Sports Medicine, vol. 33, no. 12, pp. 889-919, 2003.

[21] W. Langewitz, H. Rüddel, and H. Schächinger, "Reduced parasympathetic cardiac control in patients with hypertension at rest and under mental stress," American Heart Journal, vol. 127, no. 1, pp. 122-128, 1994.
[22] M. Morino, C. Masaki, Y. Seo et al., "Non-randomized controlled prospective study on perioperative levels of stress and dysautonomia during dental implant surgery," Journal of Prosthodontic Research, vol. 58, no. 3, pp. 177-183, 2014.

[23] K. Matsumura, K. Miura, Y. Takata et al., "Changes in blood pressure and heart rate variability during dental surgery," American Journal of Hypertension, vol. 11, no. 11, pp. 1376-1380, 1998.

[24] M. D. R. Santana, A. C. A. Sousa, E. V. Assis, L. C. Abreu, and V. E. Valenti, "Changes in heart period during endodontic treatment," Experimental \& Clinical Cardiology, vol. 20, no. 8, pp. 3389-3398, 2014.

[25] J. A. do Amaral, H. L. Guida, F. M. Vanderlei, D. M. Garner, L. C. de Abreu, and V. E. Valenti, "The effects of musical auditory stimulation of different intensities on geometric indices of heart rate variability," Alternative Therapies in Health and Medicine, vol. 21, no. 5, pp. 16-23, 2015.

[26] S. A. F. da Silva, H. L. Guida, A. M. D. S. Antonio et al., "Acute auditory stimulation with different styles of music influences cardiac autonomic regulation in men," International Cardiovascular Research Journal, vol. 8, no. 3, pp. 105-110, 2014.

[27] F. Riganello, M. D. Cortese, F. Arcuri, M. Quintieri, and G. Dolce, "How can music influence the autonomic nervous system response in patients with severe disorder of consciousness?" Frontiers in Neuroscience, vol. 9, article no. 461, 2015.

[28] M. Iwanaga, A. Kobayashi, and C. Kawasaki, "Heart rate variability with repetitive exposure to music," Biological Psychology, vol. 70, no. 1, pp. 61-66, 2005.

[29] M. Orini, R. Bailón, R. Enk, S. Koelsch, L. Mainardi, and P. Laguna, "A method for continuously assessing the autonomic response to music-induced emotions through HRV analysis," Medical and Biological Engineering and Computing, vol. 48, no. 5, pp. 423-433, 2010.

[30] S. A. F. Da Silva, H. L. Guida, A. M. Dos Santosantônio et al., "Auditory stimulation with music influences the geometric indices of heart rate variability in men," International Archives of Medicine, vol. 7, no. 1, article 27, 2014.

[31] L. L. Ferreira, L. C. M. Vanderlei, H. L. Guida et al., "Response of cardiac autonomic modulation after a single exposure to musical auditory stimulation," Noise and Health, vol. 17, no. 75, pp. 108-115, 2015.

[32] C. Mejía-Rubalcava, J. Alanís-Tavira, H. Mendieta-Zerón, and L. Sánchez-Pérez, "Changes induced by music therapy to physiologic parameters in patients with dental anxiety," Complementary Therapies in Clinical Practice, vol. 21, no. 4, pp. 282286, 2015.

[33] K. Mehr, M. Wyganowska-Swiatkowska, I. Kowalkowska, A. Kurhańska-Flisykowska, and P. Piotrowski, "Music therapy in different dental specialties," Przeglad Lekarski, vol. 69, no. 10, pp. 1049-1052, 2012.

[34] A. L. Roque, V. E. Valenti, H. L. Guida et al., "The effects of auditory stimulation with music on heart rate variability in healthy women," Clinics, vol. 68, no. 7, pp. 960-967, 2013.

[35] S. J. Lehman, J. M. Massaro, C. L. Schlett, C. J. O’Donnell, U. Hoffmann, and C. S. Fox, "Peri-aortic fat, cardiovascular disease risk factors, and aortic calcification: the Framingham Heart Study," Atherosclerosis, vol. 210, no. 2, pp. 656-661, 2010. 


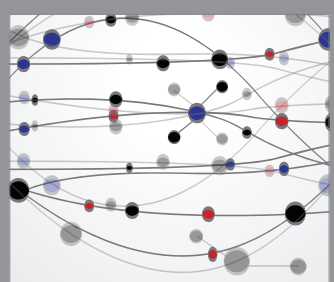

The Scientific World Journal
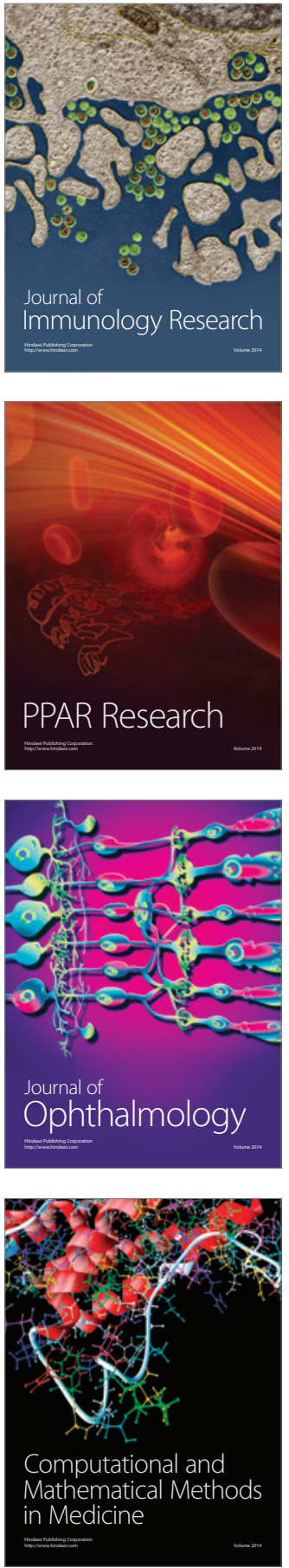

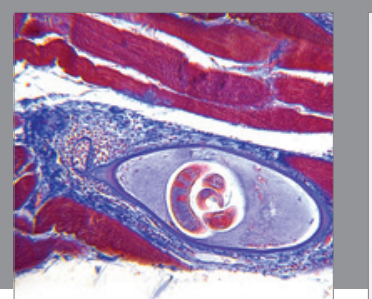

Gastroenterology Research and Practice
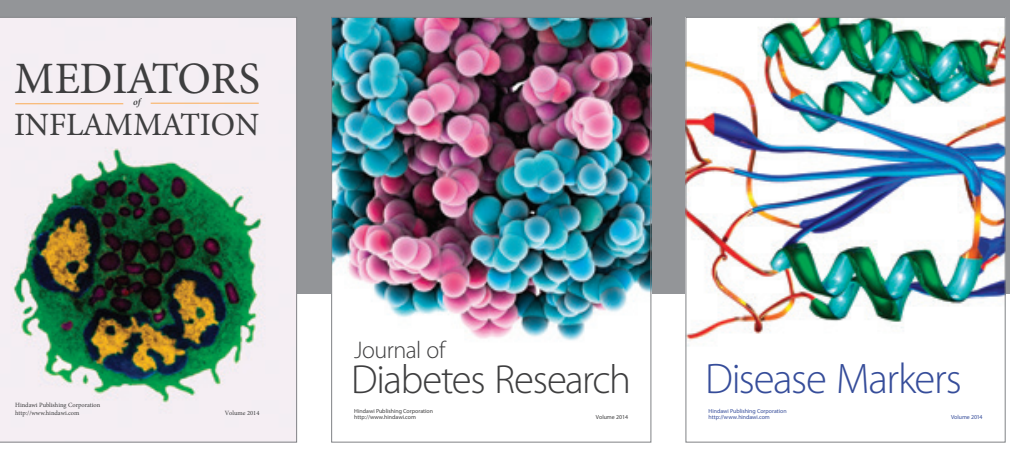

Disease Markers

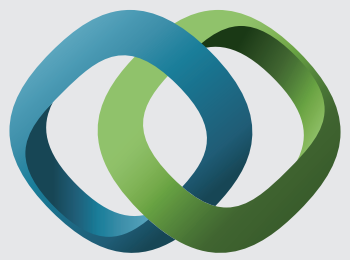

\section{Hindawi}

Submit your manuscripts at

https://www.hindawi.com
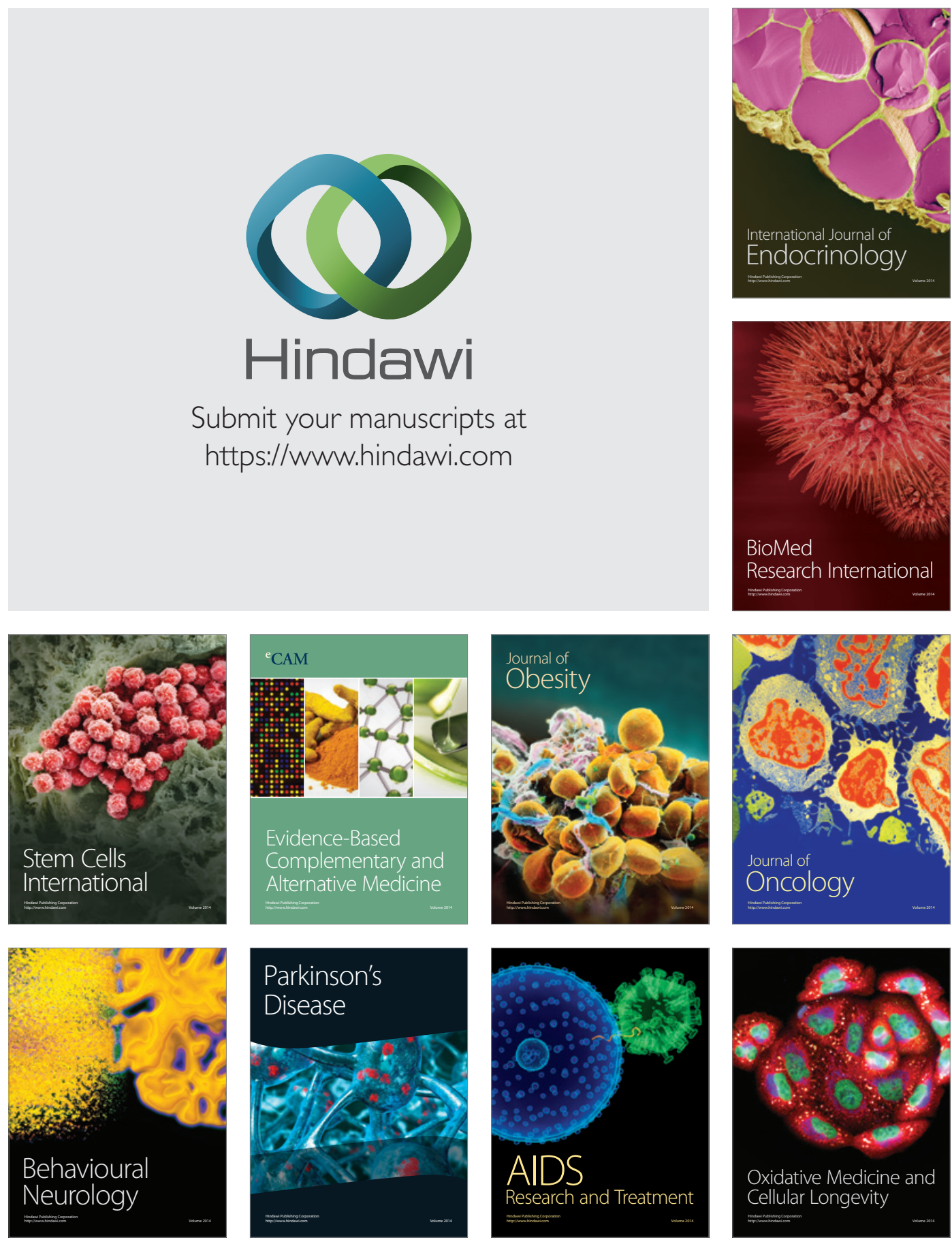